\title{
Simultaneous multi-frequency observation of the unknown redshift blazar PG 1553+113 in March-April 2008
}

\author{
J. Aleksić ${ }^{1}$, H. Anderhub ${ }^{2}$, L. A. Antonelli ${ }^{3}$, P. Antoranz ${ }^{4}$, M. Backes ${ }^{5}$, C. Baixeras ${ }^{6}$, S. Balestra ${ }^{4}$, J. A. Barrio ${ }^{4}$,
} D. Bastieri ${ }^{7}$, J. Becerra González ${ }^{8}$, J. K. Becker ${ }^{5}$, W. Bednarek ${ }^{9}$, A. Berdyugin ${ }^{10}$, K. Berger ${ }^{9}$, E. Bernardini ${ }^{11}$, A. Biland ${ }^{2}$, R. K. Bock ${ }^{12,7}$, G. Bonnoli ${ }^{13}$, P. Bordas ${ }^{14}$, D. Borla Tridon ${ }^{12}$, V. Bosch-Ramon ${ }^{14}$, D. Bose ${ }^{4}$, I. Braun ${ }^{2}$, T. Bretz ${ }^{15}$, D. Britzger ${ }^{12}$, M. Camara ${ }^{4}$, E. Carmona ${ }^{12}$, A. Carosi ${ }^{3}$, P. Colin ${ }^{12}$, S. Commichau ${ }^{2}$, J. L. Contreras ${ }^{4}$, J. Cortina ${ }^{1}$, M. T. Costado ${ }^{8,16}$, S. Covino ${ }^{3}$, F. Dazzi ${ }^{17,26}$, A. De Angelis ${ }^{17}$, E. de Cea del Pozo ${ }^{18}$, R. De los Reyes ${ }^{4,28}$, B. De Lotto ${ }^{17}$, M. De Maria ${ }^{17}$, F. De Sabata ${ }^{17}$, C. Delgado Mendez ${ }^{8,27}$, A. Domínguez $^{19}$, D. Dominis Prester ${ }^{20}$, D. Dorner ${ }^{2}$, M. Doro ${ }^{7}$, D. Elsaesser ${ }^{15}$, M. Errando ${ }^{1}$, D. Ferenc ${ }^{21}$, E. Fernández ${ }^{1}$, R. Firpo ${ }^{1}$, M. V. Fonseca ${ }^{4}$, L. Font ${ }^{6}$, N. Galante ${ }^{12}$, R. J. García López ${ }^{8,16}$, M. Garczarczyk ${ }^{1}$, M. Gaug ${ }^{8}$, N. Godinovic ${ }^{20}$, F. Goebel ${ }^{12,29}$, D. Hadasch ${ }^{18}$, A. Herrero ${ }^{8,16}$, D. Hildebrand ${ }^{2}$, D. Höhne-Mönch ${ }^{15}$, J. Hose ${ }^{12}$, D. Hrupec ${ }^{20}$, C. C. Hsu ${ }^{12}$, T. Jogler ${ }^{12}$, S. Klepser ${ }^{1}$, T. Krähenbühl ${ }^{2}$, D. Kranich ${ }^{2}$, A. La Barbera ${ }^{3}$, A. Laille ${ }^{21}$, E. Leonardo ${ }^{13}$, E. Lindfors ${ }^{10}$, S. Lombardi ${ }^{7}$, F. Longo ${ }^{17}$, M. López ${ }^{7}$, E. Lorenz ${ }^{2,12}$, P. Majumdar ${ }^{11}$, G. Maneva ${ }^{22}$, N. Mankuzhiyil ${ }^{17}$, K. Mannheim ${ }^{15}$, L. Maraschi3 ${ }^{3}$, M. Mariotti ${ }^{7}$, M. Martínez ${ }^{1}$, D. Mazin ${ }^{1}$, M. Meucci ${ }^{13}$, J. M. Miranda ${ }^{4}$, R. Mirzoyan ${ }^{12}$, H. Miyamoto ${ }^{12}$, J. Moldón ${ }^{14}$, M. Moles ${ }^{19}$, A. Moralejo ${ }^{1}$, D. Nieto ${ }^{4}$, K. Nilsson ${ }^{10}$, J. Ninkovic ${ }^{12}$, R. Orito ${ }^{12}$, I. Oya ${ }^{4}$, R. Paoletti ${ }^{13}$, J. M. Paredes ${ }^{14}$, M. Pasanen ${ }^{10}$, D. Pascoli ${ }^{7}$, F. Pauss ${ }^{2}$, R. G. Pegna ${ }^{13}$, M. A. Perez-Torres ${ }^{19}$, M. Persic ${ }^{17,23}$, L. Peruzzo ${ }^{7}$, F. Prada ${ }^{19}$, E. Prandini ${ }^{7}$, N. Puchades ${ }^{1}$, I. Puljak ${ }^{20}$, I. Reichardt ${ }^{1}$, W. Rhode ${ }^{5}$, M. Ribó ${ }^{14}$, J. Rico ${ }^{24,1}$, M. Rissi ${ }^{2}$, S. Rügamer ${ }^{15}$, A. Saggion ${ }^{7}$, T. Y. Saito ${ }^{12}$, M. Salvati ${ }^{3}$, M. Sánchez-Conde ${ }^{19}$, K. Satalecka ${ }^{11}$, V. Scalzotto ${ }^{7}$, V. Scapin ${ }^{17}$, T. Schweizer ${ }^{12}$, M. Shayduk ${ }^{12}$, S. N. Shore ${ }^{25}$, A. Sierpowska-Bartosik ${ }^{9}$, A. Sillanpää ${ }^{10}$, J. Sitarek ${ }^{12,9}$, D. Sobczynska ${ }^{9}$, F. Spanier ${ }^{15}$, S. Spiro ${ }^{3}$, A. Stamerra ${ }^{13}$, B. Steinke ${ }^{12}$, N. Strah ${ }^{5}$, J. C. Struebig ${ }^{15}$, T. Suric ${ }^{20}$, L. Takalo ${ }^{10}$, F. Tavecchio ${ }^{3}$, P. Temnikov ${ }^{22}$, D. Tescaro ${ }^{1}$, M. Teshima ${ }^{12}$, D. F. Torres ${ }^{24,18}$, N. Turini ${ }^{13}$, H. Vankov ${ }^{22}$, R. M. Wagner ${ }^{12}$, V. Zabalza ${ }^{14}$, F. Zandanel ${ }^{19}$, R. Zanin ${ }^{1}$, J. Zapatero ${ }^{6}$

(The MAGIC Collaboration)

E. Pian ${ }^{33,39}$, V. Bianchin ${ }^{30}$, F. D’Ammando ${ }^{31}$, G. Di $\operatorname{Cocco}^{30}$, D. Fugazza ${ }^{32}$, G. Ghisellini' ${ }^{32}$, O. M. Kurtanidze ${ }^{38}$, C. M. Raiteri ${ }^{34}$, G. Tosti ${ }^{35}$, A. Treves ${ }^{36}$, S. Vercellone ${ }^{37}$, and M. Villata ${ }^{34}$

(Affiliations can be found after the references)

Received 5 November 2009; accepted 3 March 2010

\section{ABSTRACT}

The blazar PG 1553+113 is a well known TeV $\gamma$-ray emitter. In this paper we determine its spectral energy distribution through simultaneous multi-frequency data to study its emission processes. An extensive campaign was carried out between March and April 2008, where optical, X-ray, high-energy (HE) $\gamma$-ray, and very-high-energy (VHE) $\gamma$-ray data were obtained with the KVA, Abastumani, REM, RossiXTE/ASM, AGILE and MAGIC telescopes, respectively. We combine the data to derive the source's spectral energy distribution and interpret its double-peaked shape within the framework of a synchrotron self-Compton model.

Key words. BL Lacertae objects: individual: PG 1553+113 - Gamma rays: observation - Gamma rays: theory

\section{Introduction}

The transformation of gravitational energy in an accretion disk around a supermassive black hole into radiation is the widely believed underlying cause of emission in active galactic nuclei (AGN). Furthermore, the emission is beamed from the jet perpendicular to the disk by a mechanism that although not fully understood yet, most likely relates to the focusing properties of the rotating, fully ionized accretion disk (e.g. Blandford \& Znajek 1977). The viewing angle of the observer determines the

Send offprint requests to: N. Mankuzhiyil, e-mail: nijil.mankuzhiyil@uniud.it observed phenomenology of AGN (Urry \& Padovani 1995). The AGN whose relativistic plasma jets point towards the observer are called blazars. The blazar class includes flat spectrum radio quasars (FSRQs) and BL Lac objects, the main difference between the two classes is in their emission lines, which are strong and quasar-like for FSRQs and weak or absent in BL Lacs.

The overall (radio-to- $\gamma$-ray) spectral energy distribution (SED) of blazars shows two broad non-thermal continuum peaks. For high-peaked BL Lac objects (HBLs), the first peak of the SED is in the UV/X-ray bands [as opposed to IR/optical for low peaked BL Lac objects, LBLs], whereas the second peak is in the multi-GeV band (multi MeV for LBLs). The lowenergy peak is thought to arise from electron synchrotron emis- 
sion. The leptonic model sugests that the second peak forms due to inverse Compton emission. This can be due to scattering of the synchrotron photons by the non-thermal population of electrons responsible for the synchrotron radiation (synchrotron self-Compton, SSC; eg: Maraschi 1992) or of photons that originate outside the relativistic plasma blob (external Compton, EC; an external source of these 'seed'photons could be the accretion disk (eg: Dermer 1993) and/or the broadline region (eg: Sikora et al. 1994)). Blazars often show violent flux variability, which may or may not be correlated between the different energy bands. Strictly simultaneous observations are crucial to investigate these correlations and understand the underlying physics of blazars.

The HBL source PG 1553+113 was firmly detected at very high-energy $\gamma$-rays (VHE; photon energy $\mathrm{E}>100 \mathrm{GeV}$ ) by the MAGIC telescope at a significance level of $8.8 \sigma$ above $200 \mathrm{GeV}$, based on data from April - May 2005 and January - April 2006 (Albert et al. 2007). Observations with the H.E.S.S. telescope array in 2005 yielded a tentative detection in the VHE band, at the level of $4 \sigma(5.3 \sigma$ using a low energy threshold analysis; Aharonian et al. 2006), which was confirmed later with the combination of the 2005 and 2006 datasets (Aharonian et al. 2008). After the first detection of PG 1553+113 with MAGIC, a multifrequency campaign on this source was conducted in July 2006 (Albert et al. 2009). The main difference between our present and the previous campaign is the use of X-ray and the highenergy (HE; photon energy $\mathrm{E}>100 \mathrm{MeV}$ ) flux.

The lack of detection of spectral lines (neither in emission nor in absorption) in the optical spectrum of PG $1553+113$ makes it impossible to directly measure its redshift (Falomo \& Treves 1990). However, an ESO-VLT spectroscopic survey of unknown-redshift BL Lac objects suggests $z>0.09$ (Sbarufatti et al. 2006), while the absence of host galaxy detection in HST images raises this lower limit to $z>0.25$ (Treves et al. 2007). On the other hand, the absence of a break in the VHE spectrum can be interpreted as suggesting $z<0.42$ (Mazin \& Goebel 2007). The absence of a Ly- $\alpha$ forest (Falomo \& Treves) in the the spectrum also constrains a lower redshift. The data obtained in the far-UV by the Cosmic Origins Spectrograph installed in the Hubble Space Telescope is of sufficient quality to select $\sim 40$ Ly- $\alpha$ absorbers at low redshift including a strong line at $\mathrm{z}=0.395$, which constrains the resdshift of the source to be $z>0.395$ (Danforth, private comm.).

\section{Optical and near infrared (NIR) data}

\subsection{Kungliga Vetenskapsakademien observations}

The Kungliga Vetenskapsakademien (KVA, Royal Swedish Academy of Sciences) telescope is located at the Roque de los Muchachos, in the North-Atlantic canary islands of La Palma and is operated by the Tuorla Observatory. The telescope is composed of a $0.6 \mathrm{~m} \mathrm{f} / 15$ Cassegrain devoted to polarimetry, and a $0.35 \mathrm{~m} \mathrm{f} / 11 \mathrm{SCT}$ auxiliary telescope for multicolor photometry. This telescope has been successfully operated remotely since the fall 2003. The KVA is used for optical (R-band) support observations during MAGIC observations. Typically, one measurement per night and per source is conducted. Photometric measurements are made in differential mode, i.e. by obtaining CCD images of the target and calibrated comparison stars in the same field of view (Fiorucci \& Tosti 1996; Fiorucci et al. 1998; Villata et al. 1998).

\subsection{Abastumani observations}

Observations at the Abastumani Observatory (Georgia, FSU) were performed with the $70 \mathrm{~cm}$ meniscus telescope (f/3). This is equipped with an Apogee Ap6E CCD camera, with $390 \times 390$ pixels, and a field of view of $15 \times 15$ arcmin. Its quantum efficiency is $40 \%$ at $4000 \AA$ and $65 \%$ at $6750 \AA$. The frames were acquired in the Cousins' $R$ band and were reduced with the DAOPHOT II package 1 .

The source magnitude was derived from differential photometry with respect to a reference star in the same field, which lies $\sim 46$ arcsec east and $\sim 5$ arcsec south of PG 1553+113. According to the USNO 2.0 Catalogue (Monet et al. 1998), its magnitude is $R=13.2$.

\subsection{Rapid Eye Mount observations}

The Rapid Eye Mount (REM, a fast-slewing robotized infrared telescope: Covino et al. 2001) acquired photometry of PG $1553+113$ on 2008 April 18, 25 and May 2 with all available filters (VRIJHK). The data reduction followed standard procedures (see e.g. Dolcini et al. 2005). The mean flux of the observation is reported in Table 3. The NIR magnitudes were calibrated against the 2MASS catalog. For the SED reconstruction, all magnitudes were dereddened with the dust IR maps (Schlegel et al. 1998).

\section{X-rays: Rossi X-ray Timing Explorer / All Sky Monitor observations}

The All Sky Monitor (ASM) on board the Rossi X-ray Timing Explorer (RXTE) satellite consists of three wide angle scanning shadow cameras. The cameras, mounted on a rotating drive assembly, can cover almost $70 \%$ of the sky every 1.5 hours (Levine et al. 1996). The measurements were done between 2008 March 1 and May 31. The mean measured flux of PG $1553+113$ is shown in Table 3.

\section{4. $\gamma$-ray data}

\subsection{HE band: AGILE observations}

The Gamma-ray Imaging Detector (GRID, $30 \mathrm{MeV}$ - 30 $\mathrm{GeV}$ ) on board the high-energy astrophysics satellite AGILE (Astro-rivelatore Gamma a Immagini LEggero; Tavani et al. 2009) observed PG $1553+113$ in three different time intervals: 2008 March 16-21, March 25-30 and April 10-30. The GRID data were analyzed using the AGILE standard pipeline (see Vercellone et al. 2008 for a detailed description of the AGILE data reduction), with a bin size of $0.25^{\circ} \times 0.25^{\circ}$ for $\mathrm{E}>100$ $\mathrm{MeV}$. Only events flagged as confirmed $\gamma$-rays and not recorded while the satellite crossed the South Atlantic Anomaly were accepted. We also rejected all events with a reconstructed direction within $10^{\circ}$ from the Earth's limb, thus reducing contamination from Earth's $\gamma$-ray albedo. The source, observed at about 50 degrees off-axis with respect to the boresight, was not detected by the GRID at a significance level $>3 \sigma$, and therefore the $95 \%$ confidence level upper limit was calculated. Considering that AGILE has a higher particle background at very high offaxis angles, we calculated also the upper limit selecting only photons with energies greater than $200 \mathrm{MeV}$ to minimize the possible contamination at low energies. The log of the AGILE

\footnotetext{
${ }^{1}$ http://www.star.bris.ac.uk/ mbt/daophot/
} 
observations and the results of the analysis are reported in Table 2. During 2008 March-April the source was outside the field of view of SuperAGILE, the hard X-ray (20-60 keV) imager onboard AGILE (Feroci et al. 2007).

\subsection{Very High Energy band: MAGIC observations}

The MAGIC Telescope (Baixeras et al. 2004, Cortina et al. 2005) is the most recent generation Imaging Atmospheric Cherenkov Telescope (IACT) at La Palma, Canary Islands, Spain $\left(28.3^{\circ} \mathrm{N}\right.$, $17.8^{\circ} \mathrm{W}, 2240 \mathrm{~m}$ a.s.1.). Because of its low trigger threshold of $50 \mathrm{GeV}(25 \mathrm{GeV}$ with a special trigger set up; Albert et al. 2008a), MAGIC is well-suited for multi-frequency observations together with the instruments operating in the GeV range. The parabolic mirror dish with a total mirror area of $236 \mathrm{~m}^{2}$ allows MAGIC to collect Cherenkov light from particle showers initiated by $\gamma$-rays or other particles in the atmosphere. This Cherenkov light is focused onto a multi-pixel camera composed of 577 ultra-sensitive photomultipliers. The total field of view of the camera is $3.5^{\circ}$. The incident light pulses are converted into optical signals and transmitted via optical fiber to a twolevel trigger system. The selected events are digitized by $2 \mathrm{GHz}$ Flash ADCs (Goebel et al. 2007). With a statistical analysis of the recorded light distribution and the orientation of the shower image in the camera, the energy of the primary particle and its incoming direction are reconstructed.

The MAGIC observations for this campaign were carried out on 2008 March 16-18 and April 13, 28-30. The zenith angle of the data set ranges from 18 degrees to 36 degrees. Observations were performed in wobble mode (Fomin et al. 1994), where the object was observed at 0.4 degree offset from the camera center in opposite directions every 20 minutes. After data rejection based on the standard quality cuts and the trigger rate, 7.18 hours of total effective observation time data were selected.

An automatic analysis pipeline (Dorner at al., 2005, Bretz \& Dorner, 2008) was used to process the data, which include the muon calibration (Goebel et al. 2005) and an absolute mispointing correction (Riegel et al. 2005). The charge distribution and arrival time information of the pulses of neighboring pixels was used to suppress the contribution from the night sky background in the shower images (Aliu et al. 2009). Three OFF regions were used to determine the background, providing a scaling factor of $1 / 3$ for the background calculation. The shape and orientation of the shower images were used to discriminate $\gamma$-like events from the overwhelming background. To select the $\gamma$-like events a dynamical cut in Area (Area $=\pi \cdot$ WIDTH·LENGTH) versus SIZE (total charge contained in an image) and a cut in $\vartheta$ (angular distance between real source position and reconstructed source position) were applied. More details on the cuts can be found in Riegel \& Bretz (2005), and the above mentioned image parameters are described by Hillas (1985). The reconstructed $\gamma$ ray spectrum is shown in Fig.1. For the spectral reconstruction, looser cuts were applied to ensure that more than $90 \%$ of the simulated gamma photons survived. Varying cut efficiencies between $50 \%$ and $95 \%$ over the entire energy range were applied to the data to check systematic effects of the cut efficiency on the spectral shape (shown as gray area in Fig.1). Data which were affected by calima (sand dust from the Sahara in an air layer between $1.5 \mathrm{~km}$ and $5.5 \mathrm{~km}$ a.s.l. causing absorption of the Cherenkov light) were corrected following the method described in Dorner et al. (2009).

\begin{tabular}{|l|c|c|}
\hline Observation period & $F_{0}\left[\mathrm{ph} \mathrm{TeV}^{-1} \mathrm{~s}^{-1} \mathrm{~m}^{-2}\right]$ & $\Gamma$ \\
\hline March-April 2008 & $2.0 \pm 0.3 \times 10^{-6}$ & $-3.4 \pm 0.1$ \\
\hline March 2008 & $1.9 \pm 0.4 \times 10^{-6}$ & $-3.5 \pm 0.2$ \\
\hline April 2008 & $2.1 \pm 0.4 \times 10^{-6}$ & $-3.3 \pm 0.2$ \\
\hline $\begin{array}{l}\text { April-May 2005 + } \\
\text { January-April 2006 }\end{array}$ & $1.8 \pm 0.3 \times 10^{-6}$ & $-4.2 \pm 0.3$ \\
\hline
\end{tabular}

Table 1. $F_{0}$ and $\Gamma$ during the MAGIC current observations and the previous observation. The errors are statistical only. The systematic uncertainty is estimated to be $35 \%$ in the flux level and 0.2 in the photon index (Albert et al. 2008a).

\section{Results and Discussion}

Analyzing the MAGIC data, an excess of $415 \gamma$-like events, over 1835 normalized background events was found, yielding a significance of $8.0 \sigma$. The resulting differential VHE spectrum of PG $1553+113$ averaged over all observing intervals is plotted in Fig.1 (filled circles). It can be described by a power law $\frac{d N}{d E}=F_{0}\left(\frac{E}{200 \mathrm{GeV}}\right)^{\Gamma} \mathrm{m}^{-2} \mathrm{~s}^{-1} \mathrm{TeV}^{-1}$, where $F_{0}$ is the normalization flux at $200 \mathrm{GeV}$ and $\Gamma$ is the photon index during our observation, which are both given in Table 1 . The test on a possible spectral cut-off was also performed. However, fewer points of the spectrum do not favour a cut-off power law over a simple power law. The lowest point of the spectrum is at $82 \mathrm{GeV}$, mainly because of the losses of low-energy events from the cleaning and $\gamma$-selection cuts. The values obtained during our previous observations (Albert et al. 2007) are also given in Table 1.

The interaction of VHE $\gamma$-rays with the extragalactic background light (EBL; a recent review can be found in Mazin $\&$ Raue 2007) leads to an attenuation of the VHE $\gamma$-ray flux via $e^{+} / e^{-}$pair production. We computed the de-absorbed (i.e., intrinsic) fluxes with a specific 'low star formation model'of the EBL (Kneiske et al. 2004), assuming a source redshift of $z=0.3$. The resulting de-absorbed points are represented as empty squares in Fig.1.

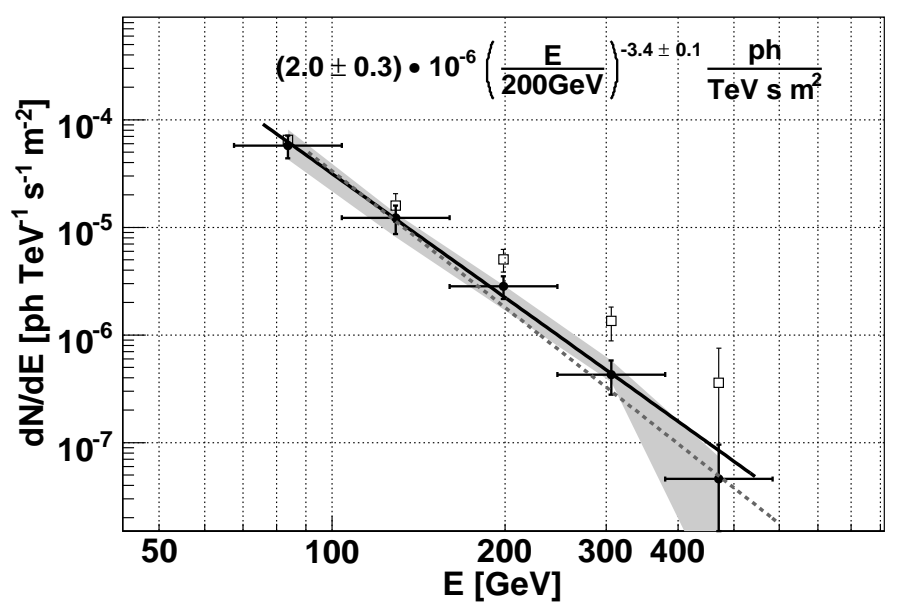

Fig. 1. MAGIC measured spectrum of PG 1553+113 (filled circles). The statistical significance of the points from left to right are 2.7, 4.2, 3.4, 4.3, and 3.0 $\sigma$ respectively. The EBL-corrected points are shown as empty squares. The spectrum obtained during our first observation is shown as a dashed line.

The HE data reduction results from AGILE are summarized in Table 2. The $2 \sigma$ upper limits obtained by AGILE are con- 
sistent with the average flux point observed by the Fermi-LAT for this source during 2008 August-Octorber (Abdo et al. 2009). The upper limit obtained in the third time interval was used for the modeling of the SED. The fluxes and corresponding effective photon frequencies of the other telescopes which contribute to this multi-frequency campaign are reported in Table 3.

\begin{tabular}{|c|c|c|}
\hline Time interval & Energy & U.L.Flux $\left[\mathrm{ph} \mathrm{m}^{-2} \mathrm{~s}^{-1}\right]$ \\
\hline \multirow{2}{*}{ March 16-21 } & $>100 \mathrm{MeV}$ & $5.6 \times 10^{-3}$ \\
& $>200 \mathrm{MeV}$ & $3.6 \times 10^{-3}$ \\
\hline \multirow{2}{*}{ March 25-30 } & $>100 \mathrm{MeV}$ & $5.5 \times 10^{-3}$ \\
& $>200 \mathrm{MeV}$ & $2.8 \times 10^{-3}$ \\
\hline \multirow{2}{*}{ April 10-30 } & $>100 \mathrm{MeV}$ & $3.4 \times 10^{-3}$ \\
& $>200 \mathrm{MeV}$ & $2.1 \times 10^{-3}$ \\
\hline
\end{tabular}

Table 2. $2 \sigma$ Upper limit calculated from the AGILE data in three different time intervals.

The SED of PG $1553+113$ is shown in Fig.2. The VHE and HE $\gamma$-ray flux points are from MAGIC and AGILE respectively. The X-ray point, provided by $R \mathrm{XTE} / \mathrm{ASM}$, represents the average flux between March 1 and May 31, 2008. The optical R-band point, provided by the KVA telescope, is the average flux obtained on 2008 March 18 and 19. The flux provided by Abastumani is the average flux of the 2008 April 1 - May 17 observations. In addition to these data we also used the NIR flux from REM. To assess the soundness of this addition, we checked the optical variability of the source during this period using Abastumani data, and found that the source was essentially stable (minimum and maximum values of $\log \left(v F_{v}\right)$ are -10.14 and -10.02 respectively). For a comparison of the HE flux, we included the flux points from the Fermi $\gamma$-ray Space Telescope (Flux, $F(E>100 \mathrm{MeV})=8 \pm 1 \times 10^{-4} \mathrm{ph} \mathrm{m}^{-2} \mathrm{~s}^{-1}$ and photon index, $\Gamma=1.7 \pm 0.6$; Abdo et al. 2009). The average flux $(15-30 \mathrm{keV})$ obtained from the X-ray satellite $S$ wift/BAT during 39 months (2004 December - 2008 February) of observation (Cusumano et al. 2010) is also included.

We fitted the resulting simultaneous SED with a homogeneous one-zone SSC model (Tavecchio et al. 2001). The model assumes that the source is a spherical plasmon of a radius $\mathrm{R}$, moving with a Doppler factor $\delta$ towards the observer at an angle $\theta$ with respect to the line of sight threaded with a uniforming distributed tangled magnetic field of the strength $B$. The injected relativistic particle population is described as a broken powerlaw spectrum with the normalization $K$, extending from $\gamma_{\text {min }}$ to $\gamma_{\max }$ with the indices $n_{1}$ and $n_{2}$ below and above the break Lorentz factor $\gamma_{\mathrm{br}}$. By fitting the observed flux with the model, we obtained the following parameters: $\gamma_{\min }=1, \gamma_{\mathrm{br}}=3 \times 10^{4}$, $\gamma_{\max }=2 \times 10^{5}, K=0.5 \times 10^{4} \mathrm{~cm}^{-} 3, n_{1}=2, n_{2}=4.7, B=0.7 \mathrm{G}$, $R=1.3 \times 10^{16} \mathrm{~cm}$, and $\delta=23$. The optical and X-ray flux constrain on the slope of the electron energy distribution (EED), while the X-ray and VHE spectrum fix the Lorentz factors.

The difference between the current SED and the previous one published in Albert et al. (2007) is due to flux variation in the X-ray and a small variation of the slope of VHE spectrum. We fitted the previous result with the Tavecchio et al. (2001) SSC model to compare the physical parameters of the SED. The difference arises from the EED, but the slopes and $\gamma_{\max }$ remain constant. The $\gamma_{\min }$ and $\gamma_{\mathrm{br}}$ of the previous observation are found to be $3 \times 10^{3}$ and $2.7 \times 10^{4}$ respectively.

During this campaign, no significant variability of the VHE flux is found. The integral flux $(\mathrm{E}>200 \mathrm{GeV})$ during these observations is $1.3 \pm 0.3 \times 10^{-7} \mathrm{~cm}^{-2} \mathrm{~s}^{-1}$ while during the first observa-

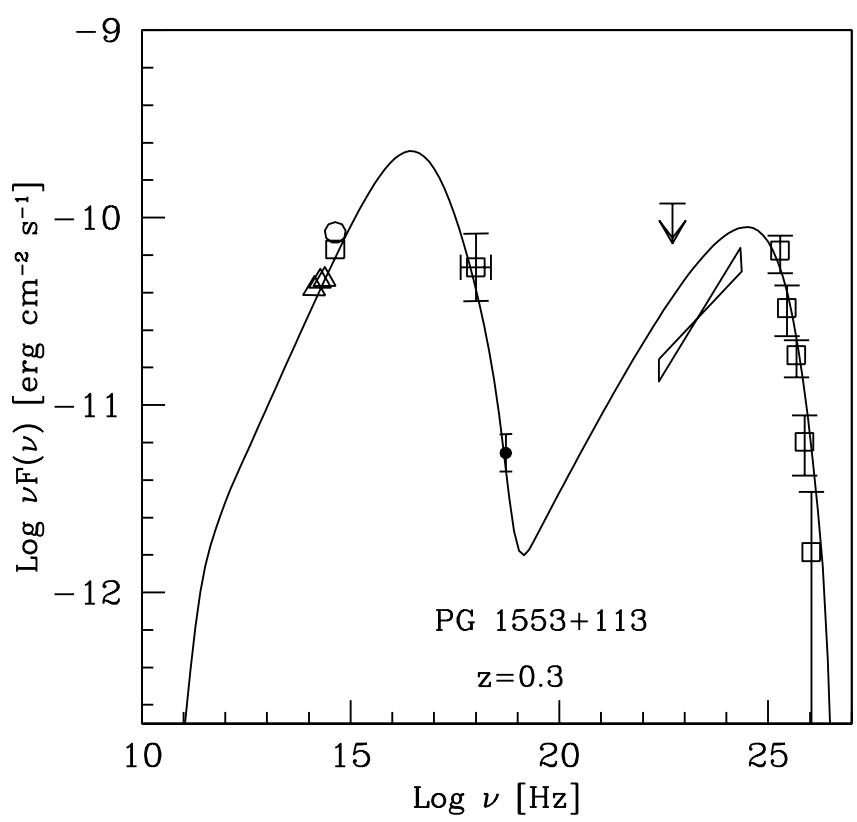

Fig. 2. Average SED of PG 1553+113 measured in 2008 MarchApril. The empty triangles denote the REM data, the open square represents the KVA data, the open circle denotes the Abastumani data, and the open square denotes RXTE/ASM data. The arrow at HE denotes the AGILE upper limit. The empty squares in the VHE range are the de-absorbed MAGIC data. We also show the non-simultaneous flux points from Fermi (bowtie) and $S$ wift/BAT (small filled circle).

\begin{tabular}{|c|c|c|}
\hline Instrument & $\log (v[\mathrm{~Hz}])$ & $\log (v \mathrm{~F}(v))\left[\mathrm{erg} \mathrm{cm}^{-2} \mathrm{~s}^{-1}\right]$ \\
\hline KVA & 14.63 & -10.17 \\
\hline Abastumani & 14.63 & -10.08 \\
\hline & 14.38 & -10.33 \\
REM & 14.27 & -10.34 \\
& 14.13 & -10.38 \\
\hline XTE & 18.03 & -10.3 \\
\hline
\end{tabular}

Table 3. Effective frequencies and corresponding fluxes from PG 1553+113 from KVA, Abastumani, REM and RXTE instruments obtained during this campaign.

tions it was $1.0 \pm 0.4 \times 10^{-7} \mathrm{~cm}^{-2} \mathrm{~s}^{-1}$. The X-ray flux ${ }^{2}$ increases by about a factor of two, while the averaged X-ray flux during 39 months of $S$ wift/BAT observations agrees with our SED. The optical flux during our first observation and the current observation does not show any significant variability. The Fermi bowtie and lowest-energy MAGIC data points together with the model fit indicate a variability at $\mathrm{HE}$ or VHE $\gamma$-rays.

Our results suggest that the variability of PG $1553+113$ at different frequencies is time-dependent: hence only a simultaneous multi-frequency monitoring campaign over a large time span will give more information on the source. Relative to this it is worth mentioning that the AGILE and MAGIC data presented here constitute the first simultaneous broad-band $\gamma$-ray observation (and ensuing SED) of any blazar, though the first simultaneous detection accomplished during the multi-frequency

\footnotetext{
2 Note that the X-ray data used in Albert et al. (2007) was not taken simultaneously with VHE and optical data.
} 
campaign of Mkn 421 (Donnarumma et al. 2009), and the first broad-band $\gamma$-ray spectrum was obtained from PKS 2155-304 (Aharonian et al. 2009) by H.E.S.S. and Fermi.

Acknowledgements. The MAGIC collaboration would like to thank the Instituto de Astrofísica de Canarias for the excellent working condition at the Observatorio del Roque de los Muchachos at La Palma. Major support from Germany's Bundesministerium für Bildung, Wissenschaft, Forschung und Technologie and Max-Planck-Gesellschaft, Italy's Istituto Nazionale di Fisica Nucleare (INFN) and Istituto Nazionale di Astrofisica (INAF), and Spain's Ministerio de Ciencia e Innovacion is gratefully acknowledged. The work was also supported by Switzerland's ETH Research grant TH34/043, Poland's Ministertwo Nauki i Szkolnictwa Wyższego grant N N203 390834 , and Germany's Young Investigator Program of the Helmholtz Gemeinschaft. This work was also supported by Georgian National Science Foundation grant GNSF/ST07/4-180. EP acknowledges support from the Italian Space Agency through grants ASI-INAF I/023/05/0 and ASI I/088/06/0. N.M. would like to thank to C.W. Danforth for the private communication regarding the newly estimated redshift of the source.

\section{References}

Abdo, A. A., Ackermann, M., Ajello, M. et al. 2009, ApJ, 700, 597 Aharonian, F. A.,Akhperjanian, A. G., Bazer-Bachi, A. R. et al. 2006, A\&A 448, L19

Aharonian, F. A., Akhperjanian, A. G., Barres de Almeida, U. et al. 2008, A\&A, 477, 481

Aharonian, F. A., Akhperjanian, A. G., Anton, G. et al. 2009, ApJ, 696, L150

Albert, J., Aliu, E., Anderhub, H. et al. 2007, ApJ, 654, 119

Albert, J., Aliu, E., Anderhub, H. et al. 2008a, ApJ, 674, 1037

Albert, J., Aliu, E., Anderhub, H. et al. 2008b, Nucl. Instr. Methods A, 588 , 424

Albert, J., Aliu, E., Anderhub, H. et al. 2009, A\&A, 493, 467

Aliu, E., Anderhub, H., Antonelli, L. A. et al. 2009, Astropart. Phys., 30, 293

Baixeras, C., Bastieri, D., Bigongiari, C. et al. 2004, Nucl. Instrum. Methods A, 518, 188

Blandford, R. D. \& Znajek, R. L. 1997, MNRAS, 179, 433

Bretz, T. for the MAGIC collaboration, 2005, Proc. 29th ICRC, 4, 315

Bretz, T. \& Dorner, D. 2008, AIP Conf. Proc., 1085, 664

Cortina, J., Armada, A., Biland, A. et al. for the MAGIC collaboration, 2005 , Proc. 29th ICRC, 5, 359

Covino, S., Zerbi, F., Chincarini, G. et al. 2001, ASP Conference Series, Vol 277

Cusumano, G., La Parola, V., Segreto, A. et al. 2009, A\&A, 510, A48

Dermer, C.D. \& Schlickeiser, A. 1993, ApJ, 416, 458

Dolcini, A., Covino, S., Treves, A. et al. 2005, A\&A, 443, L33

Donnarumma, I., Vittorini, V., Vercellone, S. et al. 2009, ApJ, 691, L13

Dorner, D., Bretz, T., Berger, K. for the MAGIC collaboration, 2005, Proc. 29th

ICRC, 5, 175

Dorner, D., Nilsson, K., Bretz, T. 2009, A\&A arXiv:0808.0279

Falomo, R. \& Treves, A. 1990, PASP , 102, 1120

Feroci, M.,Costa, E., Soffitta. P. et al. 2007, Nucl. Instrum. Methods Phys. Res. A, 581, 728

Fomin, V. P., Fennell, S., Punch, M. et al. 1994, Astropart. Phys. 2, 151

Fiorucci, M. \& Tosti, G. 1996, A\&AS , 116, 403

Fiorucci, M. Tosti, G. \& Rizzi, N. 1998, PASP, 110, 105

Goebel, F., Mase, K., Meyer, M. et al. for the MAGIC collaboration, 2005,

Proc. 29th ICRC, 5, 179

Goebel, F., Bartko, H., Carmona, E. et al. 2007, Proc. 30th ICRC, 3, 1481

Hillas, A.M. 1985, Proc. 19th ICRC, 3, 445

Kneiske, T. M., Bretz, T., Mannheim, K. \& Hartmann, D. H. 2004, A\&A, 413, 807

Levine, A. M., Bradt, H., Cui, W. et al. 1996, ApJ, 469, L33

Maraschi, L. Ghisellini, G. \& Celotti, A. 1992, ApJ, 397, L5

Mazin, D. \& Goebel, F. 2007, ApJ, 655, L13

Mazin, D. \& Raue, M. 2007, A\&A, 471, 439

Monet, D., Bird, A., Canzian, B. et al. 1998, The PMM USNO-A2.0 Catalog

(http://adsabs.harvard.edu/abs/1998USNO2.C......0M

Riegel, B. \& Bretz, T. 2005, Proc. 29th ICRC, 5, 215

Riegel, B., Bretz, T., Dorner, D. \& Wagner, R. M. for the MAGIC collaboration,

2005, Proc. 29th ICRC, 5, 219

Sbarufatti, B., Treves, A., Falomo, R. et al. 2006, ApJ, 132, 1

Schlegel, D.J. 1998, ApJ, 500, 525

Sikora, M., Begelman, M. C. \& Rees, M. J. 1994, ApJ, 421, 153

Urry, C.M. \& Padovani, P. 1995, PASP, 107, 803

Tavani, M., Barbiellini, G., Argan, A., et al. 2009, A\&A, 502, 995

Treves, A., Falomo, R., \& Uslenghi, M. 2007, A\&A, 473, L17
Tavecchio, F., Maraschi, L., \& Pian, E. et al. 2001, ApJ, 554, 725

Vercellone, S., Chen, A. W., Giuliani, A. et al. 2008, ApJ, 676, L13

Villata, M., Raiteri, C. M., Lanteri, L., Sobrito, G. \& Cavallone, M. 1998, A\&AS, 130, 305

1 IFAE, Edifici Cn., Campus UAB, E-08193 Bellaterra, Spain

2 ETH Zurich, CH-8093 Switzerland

3 INAF National Institute for Astrophysics, I-00136 Rome, Italy

4 Universidad Complutense, E-28040 Madrid, Spain

5 Technische Universität Dortmund, D-44221 Dortmund, Germany

6 Universitat Autònoma de Barcelona, E-08193 Bellaterra, Spain

7 Università di Padova and INFN, I-35131 Padova, Italy

8 Inst. de Astrofísica de Canarias, E-38200 La Laguna, Tenerife, Spain

9 University of Łódź, PL-90236 Lodz, Poland

10 Tuorla Observatory, University of Turku, FI-21500 Piikkiö, Finland

11 Deutsches Elektronen-Synchrotron (DESY), D-15738 Zeuthen, Germany

12 Max-Planck-Institut für Physik, D-80805 München, Germany

13 Università di Siena, and INFN Pisa, I-53100 Siena, Italy

${ }_{14}$ Universitat de Barcelona (ICC/IEEC), E-08028 Barcelona, Spain

15 Universität Würzburg, D-97074 Würzburg, Germany

16 Depto. de Astrofisica, Universidad, E-38206 La Laguna, Tenerife, Spain

17 Università di Udine, and INFN Trieste, I-33100 Udine, Italy

18 Institut de Ciències de l'Espai (IEEC-CSIC), E-08193 Bellaterra, Spain

${ }_{19}$ Inst. de Astrofísica de Andalucía (CSIC), E-18080 Granada, Spain

${ }^{20}$ Croatian MAGIC Consortium, Institute R. Boskovic, University of

Rijeka and University of Split, HR-10000 Zagreb, Croatia

21 University of California, Davis, CA-95616-8677, USA

22 Inst. for Nucl. Research and Nucl. Energy, BG-1784 Sofia, Bulgaria

${ }^{23}$ INAF/Osservatorio Astronomico and INFN, I-34143 Trieste, Italy

24 ICREA, E-08010 Barcelona, Spain

${ }^{25}$ Università di Pisa, and INFN Pisa, I-56126 Pisa, Italy

26 supported by INFN Padova

27 now at: Centro de Investigaciones Energéticas, Medioambientales

y Tecnológicas (CIEMAT), Madrid, Spain

28 now at: Max-Planck-Institut für Kernphysik, D-69029 Heidelberg, Germany

29 deceased

30 INAF/IASF-Bologna, I-40129 Bologna, Italy

31 INAF/IASF-Roma,I-00133 Roma, Italy

32 INAF, Astronomical Obs. Brera, I-23807 Merate, Italy

33 INAF, Trieste Astronomical Observatory, I-34143 Trieste, Italy

34 INAF, Astronomical Obs. Torino, I-10025 Torino, Italy

35 University of Perugia, I-06123 Perugia, Italy

${ }^{36}$ University of Insubria, I-22100 Como, Italy

37 INAF, IASF-Milano, I-20133 Milano, Italy

38 Abastumani Astrophysical Observatory, 383762 Abastumani, Georgia

39 now at: Scuola Normale Superiore, I-56126 Pisa, Italy 